\title{
Autodeterminación en personas con discapacidad intelectual y del desarrollo: revisión del concepto, su importancia y retos emergentes
}

\author{
Self-determination in persons with intellectual and developmental \\ disabilities: a revision of the construct, its importance and emerging \\ challenges
}

\section{Resumen}

Los avances en la conceptualización de la discapacidad intelectual y el auge de movimientos de autodefensa y empoderamiento han influido, indudablemente, en la comprensión e importancia de constructos como la autodeterminación. En este trabajo el objetivo central es aportar una actualización sobre la forma de entender el constructo y la importancia del desarrollo de la autodeterminación en los diferentes contextos de vida de las personas y, en concreto, de las personas con discapacidad intelectual. Igualmente, se complementa dicha actualización del estado de la cuestión mostrando los resultados obtenidos en un estudio en el que se exploraban las percepciones de las propias personas con discapacidad, sus familiares y los profesionales que trabajan con ellas sobre la importancia que otorgan a la autodeterminación. Todo ello permite poner de relieve las necesidades y retos que los profesionales y familiares se encuentran para transformar esa importancia atribuida a la autodeterminación en la creación de oportunidades para su promoción. Se discute sobre la necesidad de seguir ahondando para conocer y dar respuestas a dichos retos.

\section{Palabras clave}

Autodeterminación, discapacidad intelectual y del desarrollo, actualización, profesionales, familias.

\begin{abstract}
Advances in the conceptualization of intellectual disability and the emergence of self-advocacy movements have undoubtedly influenced constructs such as selfdetermination. In this study, an update on the way of understanding the construct is presented, as well as its importance on the development of self-determination across contexts of people with intellectual and developmental disabilities. This state of the issue is as well exemplified with the preliminary results of a study aiming to explore perceptions of self-determination importance for people with disabilities themselves, their families and the professionals who work with them. Even if professionals and families attribute high importance to self-determination, they still face needs and challenges in creating opportunities to foster self-determination in their contexts. The need to deeply explore and answer to these challenges is also discussed.
\end{abstract}

\section{Keywords}

Self-determination, intellectual and developmental disabilities, actualization, professionals, families.

\author{
Eva Vicente Sánchez \\ <evavs@unizar.es>
}

Universidad de Zaragoza. España

Cristina Mumbardó-Adam

$<$ cristinama6@blanquerna.url.edu>

Universidad Ramon Llull. España

Teresa Coma Roselló

<tcoma@unizar.es>

Universidad de Zaragoza. España

\section{Miguel Ángel Verdugo \\ Alonso}

<verdugo@usal.es>

Universidad de Salamanca. España

\section{Climent Giné Giné}

<climentgg@blanquerna.url.edu>

Universidad Ramon Llull. España

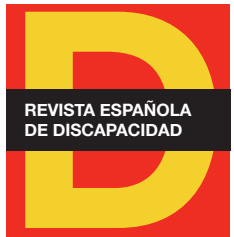

Para citar:

Vicente, E. et al. (2018):

"Autodeterminación en personas con discapacidad intelectual y del desarrollo: revisión del concepto, su importancia y retos emergentes". Revista Española de Discapacidad, 6 (II): 7-25.

Doi: <https://doi.org/10.5569/23405104.06.02.01>

Fecha de recepción: 15-02-2018 Fecha de aceptación: 26-11-2018

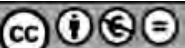




\section{Introducción}

Las últimas definiciones de discapacidad intelectual (Luckasson et al., 1992; Luckasson et al., 2002; Schalock et al., 2010) propuestas por la Asociación Americana sobre Discapacidad Intelectual y del Desarrollo (en adelante AAIDD) han tenido un impacto sustancial en la estabilización de cambios en su concepción, así como en las prácticas profesionales dirigidas a las personas con discapacidad intelectual (Verdugo y Schalock, 2010). Esta concepción socioecológica de la discapacidad intelectual proporciona un modelo de entendimiento más completo y complejo, que pone énfasis en las capacidades y potencialidades de las personas y en los apoyos que necesita para optimizar su funcionamiento individual. Paralelamente, el concepto de calidad de vida tiene igualmente un papel importante en el campo de la discapacidad intelectual como marco para el desarrollo de servicios de calidad y la evaluación de resultados personales. Para las organizaciones es fundamental trabajar la consecución de resultados personales positivos como finalidad última de la provisión de apoyos a las personas con discapacidad intelectual.

Ya en 1996, Schalock mencionó el vínculo que existe entre el actual enfoque de discapacidad intelectual y los conceptos de calidad de vida y autodeterminación cuando concluyó que "el actual paradigma de discapacidad intelectual y su estrecha relación con el énfasis en la autodeterminación, la inclusión, la equidad, el empoderamiento, los apoyos basados en la comunidad y los resultados de calidad han obligado a los proveedores de servicios a focalizarse en la mejora de la calidad de vida de las personas con discapacidad" (Schalock, 1996: 123). Con esta afirmación Schalock pretendía enfatizar que el actual concepto y paradigma de discapacidad intelectual propone que las organizaciones, entidades y más altas instituciones deben centrarse, no en compensar los déficits de la persona, sino en dirigir los esfuerzos en la provisión de apoyos individualizados que permitan a la persona participar de manera inclusiva en la comunidad, mejorar su calidad de vida, y promover su empoderamiento y su autodeterminación. De hecho, según Schalock y Verdugo (2014) para crear valor a través de la innovación en el ámbito organizacional, el concepto de calidad de vida y sus aplicaciones sitúan al individuo y/o a la familia en el centro del proceso, gracias al énfasis en la inclusión, la equidad, el desarrollo personal y la autodeterminación.

En este sentido, los recientes avances tanto en la conceptualización del constructo, como en su reconocido papel en los procesos de desarrollo de la persona y en las prácticas y servicios de las instituciones de provisión de apoyos realmente deben estar en línea y coincidir con la realidad, las demandas y las necesidades de las personas con discapacidad, de sus familias y de los profesionales de atención directa. Solo un ajuste entre dichos avances y la realidad del día a día de las personas con discapacidad y las personas con quien interactúan y se desarrollan, es decir, las personas que promueven su autodeterminación, garantiza que el contexto de la persona actúe como facilitador y no como obstáculo para actuar de forma autodeterminada. En la actualidad son pocos los datos empíricos que exploran estas perspectivas y necesidades en contexto español, aunque estos estudios resulten fundamentales para conocer los retos en los que la investigación debería profundizar.

A lo largo de este artículo el objetivo principal es presentar el estado de la cuestión en relación a la autodeterminación, abordando la importancia, las implicaciones y los nuevos retos existentes en relación a la autodeterminación de las personas con discapacidad intelectual, y haciendo un breve recorrido sobre su conceptualización teórica, sobre las dimensiones e indicadores que la definen y que destacan; y resaltando 
los posibles retos y demandas reclamados tanto por las propias personas con discapacidad intelectual, como por sus familias y los profesionales que les prestan apoyo. Este objetivo principal de carácter teórico se complementa con el análisis descriptivo de datos obtenidos sobre la importancia que atribuyen personas con discapacidad, sus familias y profesionales a aspectos concretos y observables de la autodeterminación, con el objetivo de poder, así, relacionarlo con la bibliografía existente sobre el tema. Para ello, en primer lugar, se realiza una síntesis actualizada del constructo de autodeterminación, incluyendo desde las implicaciones de los cambios en la conceptualización hasta el auge en el desarrollo de nuevos instrumentos de evaluación e intervención. De manera complementaria, se describen y analizan los resultados derivados de una de las rondas de un estudio Delphi llevado a cabo recientemente (Vicente et al., 2018) en la que una de las tareas era preguntar a personas con discapacidad intelectual, familias y profesionales sobre la importancia que atribuyen a indicadores observables de autodeterminación. Finalmente, se presentan los retos y líneas de investigación actuales que pueden dar respuesta a las necesidades relacionadas con el fomento de la autodeterminación de cada una de las partes implicadas.

\section{Actualización teórica. ¿Cómo se entiende, se define y operativiza la autodeterminación desde una perspectiva teórica?}

Varias teorías han definido el constructo de autodeterminación y su impacto en la vida de las personas con discapacidad, en la de sus familias y en la de los profesionales de atención directa. Uno de los modelos explicativos que más impacto ha tenido, y sigue teniendo, es la Teoría Funcional de Autodeterminación (Wehmeyer, 1999), que sugiere que la conducta puede definirse como autodeterminada dependiendo de la función que esta cumpla para la persona que actúa. En esta línea, la Teoría de la Agencia Causal, construida en base a la teoría funcional y las aportaciones más recientes de la psicología positiva y de la concepción socioecológica de la discapacidad presentada anteriormente, define la autodeterminación como "una característica disposicional que se pone de manifiesto cuando la persona actúa como agente causal de su propia vida" (Shogren et al., 2015: 258). Bajo este marco teórico, actuar de forma autodeterminada implica que estas acciones se puedan definir por rasgos específicos agrupados en tres dimensiones o características esenciales: acciones volitivas, acciones agenciales y creencias de control-acción.

La primera de las dimensiones implica actuar con volición, es decir, con autonomía e intencionalidad, eligiendo objetivos basados en las propias preferencias e iniciando las acciones cuándo y cómo uno escoge hacerlo. Actuar de forma volitiva es hacerlo con autonomía sin influencia indebida de terceros e iniciando las acciones cuando uno decide hacerlo (autoiniciación). La acción autodeterminada implica también ser "agencial", es decir, actuar gestionando las propias acciones para lograr determinados objetivos, monitoreando y autorregulando este proceso de consecución de objetivos. En definitiva, implica actuar dirigiendo las propias acciones hacia la consecución de objetivos personales (autodirección), regulando el proceso de acción, la toma de decisiones y la resolución de problemas en función de los obstáculos que vayan apareciendo en el proceso (autorregulación), y pensando en diferentes opciones de acción para superar los posibles obstáculos en la consecución de dichos objetivos (pensar en alternativas). Finalmente, la autodeterminación implica también mostrar creencias de control-acción, es decir, conocimiento sobre las propias 
capacidades y habilidades que se requieren para alcanzar determinados objetivos (empoderamiento), ser capaz de ajustar las expectativas a las capacidades y límites de la persona (control de las expectativas) y el sentimiento de realización personal que las acciones tienen para la persona (autorrealización). En la Tabla 1 se detallan estas características esenciales y componentes de autodeterminación definidas en la Teoría de la Agencia Causal (Shogren et al., 2015), asociadas a sus equivalentes en el marco de Teoría Funcional.

\begin{tabular}{|c|c|c|}
\hline \multicolumn{2}{|l|}{ Teoría de la Agencia Causal } & \multirow{2}{*}{ Teoría Funcional (dimensiones) } \\
\hline Dimensiones & Subdimensiones & \\
\hline Acciones Volitivas & $\begin{array}{l}\text { - Autonomía } \\
\text { - Autoiniciación }\end{array}$ & - Autonomía \\
\hline Acciones Agenciales & $\begin{array}{l}\text { - Autodirección } \\
\text { - Autorregulación } \\
\text { - Pensar en alternativas }\end{array}$ & - Autorregulación \\
\hline Creencias de Control-Acción & $\begin{array}{l}\text { - Empoderamiento } \\
\text { - } \text { Autorrealización } \\
\text { - Control de las expectativas }\end{array}$ & $\begin{array}{l}\text { - Empoderamiento } \\
\text { - Autorrealización }\end{array}$ \\
\hline
\end{tabular}

Fuente: elaboración propia (a partir de Shogren et al., 2015 y Wehmeyer, 1999).

Sin duda, la autodeterminación es un constructo complejo que cuenta con múltiples implicaciones en la vida de cualquier persona, y de manera especial en la vida de las personas con discapacidad intelectual. Tradicionalmente, se consideraba que la autodeterminación era un logro difícil de alcanzar por las personas con discapacidad intelectual, sin embargo, hoy en día se cuenta con suficientes evidencias para afirmar que la conducta autodeterminada se puede promover y desarrollar si se ponen en marcha los apoyos y las estrategias de intervención apropiadas (Algozzine et al., 2000; Cobb et al., 2009). Las iniciativas y programas diseñados para fomentar la autodeterminación se centran principalmente en trabajar una serie de habilidades y componentes específicos, observables y relacionados con la autodeterminación. Destaca la cantidad de currículos desarrollados en inglés ([Steps to Self-Determination" Curriculum] de Field y Hoffman, 1996; 2002; [Programa "Next STEP”] de Halpern et al., 2000; [The Self-Directed IEP, ChoiceMaker Curriculum] de Martin et al., 1996; [The Self-Determination Learning Model of Instruction] de Wehmeyer et al., 2000b); frente al menor número de recursos desarrollados en castellano (algunos de ellos se describen en el siguiente apartado). Múltiples investigaciones indagan también sobre la frecuencia con la que se trabajan estas habilidades en los contextos educativos como un reflejo de la importancia que se da a la autodeterminación como objetivo educativo (e.g., Wehmeyer et al., 2000a). Estos componentes (Tabla 2) subyacen a las dimensiones de autodeterminación antes descritas y se aprenden desde la infancia de manera incidental a partir de la interacción con el entorno o mediante procesos estructurados de aprendizaje (Wehmeyer et al., 1998; Wehmeyer, 2003). 


\begin{tabular}{l}
\hline Tabla 2. Componentes específicos de la conducta autodeterminada (Wehmeyer et al., 2013) \\
\begin{tabular}{|l|l|l|}
\hline Componentes específicos y observables & Autogestión y autoinstrucciones & Locus de control interno \\
\hline Tomar decisiones & $\begin{array}{l}\text { Autoobservación, auto-evaluación y } \\
\text { autorrefuerzo }\end{array}$ & $\begin{array}{l}\text { Atribuciones positivas de eficacia y } \\
\text { expectativas de control }\end{array}$ \\
\hline Hacer elecciones & $\begin{array}{l}\text { Independencias, tomar riesgos, } \\
\text { seguridad }\end{array}$ & Autoconciencia \\
\hline Solucionar problemas & Autodefensa & Autoconocimiento \\
\hline Establecimiento de metas & &
\end{tabular}
\end{tabular}

Fuente: elaboración propia (a partir de Wehmeyer et al., 2013).

\section{Evaluación e intervención en autodeterminación. ¿Qué recursos hay disponibles?}

Los recursos evaluativos constituyen un elemento fundamental en cualquier proceso de enseñanza y aprendizaje dado que permiten identificar las necesidades iniciales de la persona y guiar la toma de decisiones a lo largo de la implantación de un programa de instrucción. En este sentido, existen numerosos instrumentos de evaluación de la autodeterminación creados en contexto estadounidense (Arellano y Peralta, 2013), siendo dos de ellos desarrollados a partir de los modelos teóricos descritos previamente: la Arc's SelfDetermination Scale (Wehmeyer y Kelchner, 1995) y el Self-Determination Inventory (Shogren et al., 2017). En España, el proceso de desarrollo y validación de instrumentos de este tipo se ha desarrollado desde este mismo marco teórico, contando actualmente con distintos tipos de instrumentos. Dos de ellos se han adaptado utilizando como referencia el Modelo Funcional de Autodeterminación (Wehmeyer, 1999), en concreto: (1) la Escala ARC-INICO de evaluación de la autodeterminación, validada en adolescentes con discapacidad intelectual (Verdugo et al., 2015) y (2) la escala ARC de autodeterminación (Wehmeyer et al., 2006) fiel reflejo de la versión original en inglés. Recientemente, y acorde a la nueva reformulación del modelo basada en la Teoría de la Agencia Causal (Shogren et al., 2015), se dispone de la adaptación y validación del Inventario de Autodeterminación (Mumbardó-Adam et al., 2018), validado en adolescentes con y sin discapacidad. Todos ellos son autoinformes que debe responder el adolescente o joven, ya sea en formato autoadministrado o en formato entrevista (si la persona requiere de apoyos adicionales para su comprensión y aplicación). Este formato de aplicación, a pesar de ser imprescindible especialmente en el ámbito de la autodeterminación (no se puede hablar de la autodeterminación de una persona sin contar con ella), alberga ciertas limitaciones en su aplicación. En primer lugar, se limita la participación y evaluación de la autodeterminación de personas con discapacidad y necesidades de apoyos más significativas, o con limitaciones comunicativas graves. En este sentido, futuras líneas de investigación deberán promover la creación de instrumentos de medición de la autodeterminación adaptados a estas personas (e.g., protocolos de observación, heteroinformes,...). En segundo lugar, si bien, como hemos mencionado, hablar de autodeterminación implica necesariamente conocer la perspectiva de la persona que se está evaluando, la percepción de un informador cercano a dicha persona aporta información de innegable valía que permite contrastar la información y descubrir áreas relevantes sobre las que trabajar. Queda claro que sigue siendo necesario continuar la investigación y el 
desarrollo de instrumentos en este campo y, de hecho, existe una iniciativa en marcha para la creación (a partir del consenso entre expertos) y la validación de una escala que utilice la información proporcionada por familiares y profesionales para evaluar la autodeterminación de personas con discapacidad intelectual y no solo limitándose a adolescentes, sino expandiendo el rango de aplicación a jóvenes y adultos-jóvenes (Vicente et al., 2018).

En relación a la enseñanza y promoción de la autodeterminación, esta suele dirigirse a promover los componentes específicos y habilidades relacionadas con la autodeterminación (e.g. toma de decisiones, resolución de problemas, autogestión, autodefensa...). En este sentido, como ya se ha comentado, existe una gran cantidad de currículos de intervención disponibles en inglés. Uno de los que más se ha usado es el Self-Determined Learning Model of Instruction (Wehmeyer et al., 2000b), probablemente por la posibilidad de aplicarlo a la consecución de objetivos tanto académicos como relacionados con actividades laborales sin necesidad de enseñar las habilidades relacionadas con la autodeterminación de forma descontextualizada o poco relacionada con los objetivos y el día a día de la persona (Raley et al., en prensa). Este currículo de intervención se ha traducido y adaptado al español para su uso (Mumbardó-Adam et al., 2017) y cuenta con múltiples evidencias de su eficacia (Shogren et al., 2012; Wehmeyer et al., 2012), aunque de momento no existen datos de su uso en contexto español.

No obstante, en España disponemos de herramientas de buenas prácticas que muestran ejemplos llevados a cabo en centros para trabajar la autodeterminación. De hecho, estas herramientas de intervención no se centran exclusivamente en las personas con discapacidad. Instituciones como Plena Inclusión y otras organizaciones y entidades que trabajan por y para las personas con discapacidad intelectual fomentan iniciativas de promoción de la autodeterminación con materiales dirigidos tanto a la sensibilización de las familias de jóvenes y adultos con discapacidad intelectual (e.g. Formación en Autodeterminación para Familias; Ponce, 2010), como a los profesionales y personal de apoyo que trabajan con estas personas. El cuaderno de buenas prácticas "Autodeterminación” (Grupo de autogestores de ATZEGI, 2009) o la Guía Didáctica V.I.D.A. (Díaz et al., 2013) constituyen buenos ejemplos de guías para profesorado y el personal de apoyo sobre cómo favorecer y potenciar las oportunidades de las personas con discapacidad para practicar y desarrollar habilidades relacionadas con la autodeterminación. Si bien cabe destacar la importancia de iniciativas de esta índole dirigidas a promover la formación de familiares y profesionales en la promoción de la autodeterminación, poco sabemos sobre la importancia que familiares y profesionales atribuyen a la autodeterminación, sus dimensiones y componentes específicos. Las creencias de familiares y profesionales sobre la relevancia de promover la autodeterminación y crear oportunidades para su desarrollo tienen un impacto sobre su enseñanza (Arellano y Peralta, 2013; Wehmeyer et al., 2000a). En este sentido, dado que los familiares y profesionales de atención directa constituyen el principal apoyo para la promoción de la autodeterminación, surge la necesidad de ahondar en sus perspectivas y que a partir de ellas podamos conocer tanto las prioridades de actuación en este ámbito, como las posibles barreras y facilitadores existentes en la promoción de la autodeterminación. En definitiva, es importante explorar cómo entienden este constructo y sus implicaciones prácticas; observar si realmente consideran que es algo importante en la vida de las personas con discapacidad intelectual y detectar posibles necesidades o retos a superar en este área. En el siguiente apartado se aborda la importancia atribuida, para finalizar con el último apartado en el que se reflexiona sobre los posibles retos. 


\author{
4. Importancia de la autodeterminación. ¿Qué relevancia tiene la autodeterminación y sus \\ componentes para las personas con discapacidad intelectual, sus familiares y los profesionales que \\ trabajan con ellos?
}

Parece innegable que tener la capacidad y la oportunidad de ejercer control y ser el agente causal de nuestra propia vida es un elemento fundamental para cualquier persona. Sin embargo, en el caso de las personas con discapacidad intelectual, aunque la importancia global se puede deducir del auge de los movimientos de autodefensa y empoderamiento, sería oportuno conocer si realmente todas las partes implicadas (profesionales, familiares y las propias personas con discapacidad intelectual) compartimos la misma visión sobre aquello que consideramos más importante. Por ello, parece pertinente profundizar sobre qué componentes o indicadores son realmente importantes y si existen diferencias en la importancia que les atribuyen en función del rol que juega la persona en la vida de la persona con discapacidad. Para dar respuesta a este interrogante, en este apartado se proporcionan datos extraídos de un estudio Delphi (Vicente et al., 2018), los cuales se relacionan con la investigación previa existente a este respecto.

\title{
4.1. Contexto de la investigación
}

Los datos proporcionados forman parte de un estudio Delphi mayor, cuyo objetivo era obtener consenso entre tres paneles de expertos ( 10 profesionales, 6 familiares y 5 personas con discapacidad intelectual) sobre los indicadores más idóneos, observables e importantes para evaluar la autodeterminación a través de tres rondas iterativas (Vicente et al., 2018). La finalidad última del estudio Delphi fue obtener un pool de indicadores suficientes que reflejaran el consenso de los expertos y que representaran los elementos clave necesarios para evaluar la autodeterminación (es decir, indicadores que fueran importantes para todas las partes implicadas, que permitieran evaluar el constructo y sus dimensiones y que fueran observables).

En este artículo se analizan de manera detallada los resultados obtenidos en relación con las valoraciones hechas por los participantes sobre la importancia de los indicadores. Para ello, se extraen los datos obtenidos en una de las tareas solicitadas a los 21 expertos en la primera de las rondas de dicho estudio Delphi. Concretamente se solicitaba a todos los participantes que valoraran la importancia de un conjunto de 131 indicadores de autodeterminación, utilizando una escala de cuatro puntos (donde 1 significaba que el indicador era poco importante; y 4 que el indicador es muy importante). Se presentan los resultados de esta ronda y esta tarea específica porque los datos obtenidos reflejan bastante bien la importancia que atribuyen cada uno de los agentes implicados (personas con discapacidad, familiares y profesionales) a un conjunto de elementos o indicadores de autodeterminación suficientemente amplio.

\subsection{Descripción de los participantes}

La selección de los 21 participantes se realizó por muestreo incidental, siendo el objetivo contar con la participación de profesionales, familiares y personas con discapacidad intelectual. Los diez profesionales que participaron (ocho mujeres y dos hombres) tenían entre 26 y 56 años. Todos ellos tenían estudios universitarios y trabajaban en atención directa a personas con discapacidad intelectual o en el ámbito de 
la investigación en este campo, con más de tres años de experiencia (Media = 13.4 años). Pertenecían a distintas organizaciones y entidades de distintas comunidades autónomas de España (Aragón, Cantabria, Castilla y León, Madrid y Cataluña). Los seis familiares eran cuatro padres y dos madres de personas con discapacidad intelectual con un rango de edad entre 51 y 72 años. Sus hijos o hijas pertenecían a asociaciones y entidades de Aragón o Cantabria. Por último, participaron también cinco personas con discapacidad intelectual mayores de edad (entre 21 y 30 años) pertenecientes todos ellos a la Asociación Down Huesca. Todos ellos aceptaron libremente participar y otorgaron su consentimiento informado.

\subsection{Instrumento y procedimiento}

El desarrollo del conjunto de indicadores sometido a valoración fue desarrollado por el equipo de investigación del estudio Delphi, a partir de una fase inicial de revisión bibliográfica que permitió extraer indicadores a partir de la literatura existente y una segunda fase en la que tres investigadoras sometieron a debate y consenso esos primeros indicadores hasta llegar al pool inicial de indicadores que se utilizó en el estudio Delphi. Los 131 indicadores que formaban este pool inicial estaban agrupados en torno a las tres dimensiones del constructo (41 ítems para la dimensión acciones volitivas, 37 para acciones agenciales y 53 ítems para la dimensión creencias de control-acción). Para una mayor descripción del pool de ítems, su proceso de creación y los resultados finales obtenidos en el Delphi puede consultarse Vicente et al. (2018). Como ya se ha indicado, en este trabajo se presentan únicamente los resultados asociado a las valoraciones hechas por los participante sobre la importancia que atribuyen a los indicadores de autodeterminación.

Los expertos debían valorar el pool de 131 indicadores en función del criterio de importancia (es decir, relevancia del ítem en la vida de las personas con discapacidad intelectual y teniendo en cuenta la dimensión de autodeterminación a la que pertenecía) utilizando una escala Likert de cuatro puntos. Los participantes debían asignar a cada indicador una puntuación de 1 a 4: siendo 1 nada importante; 2 poco importante; 3 importante; y 4 muy importante. Para asignar el valor de importancia, debían recurrir a su cercanía y experiencia en el ámbito de la discapacidad (aquello que para ellos resultaba más o menos importante), no necesariamente pensando en una persona determinada, sino en su opinión general sobre cada indicador del pool.

\subsection{Resultados}

Una vez obtenidos los resultados, se analizaron a partir de estadísticos descriptivos (medias y desviaciones típicas) calculadas tanto para el total de los participantes para conocer la percepción general respecto a la autodeterminación, como por grupos para poder comparar las percepciones de unos y otros. Se realizaron también análisis no paramétricos de comparación de medias ( $u$ de Mann Whitney) para comparar la percepción de familiares y profesionales, destacando aquellos indicadores en los que se obtuvieron diferencias significativas.

En general, los datos muestran la gran importancia que tiene la autodeterminación para todos los participantes, ya que todos los indicadores (a excepción de uno) obtienen una media superior a 3, indicando que el grado de importancia atribuido en general es bastante alto. El único indicador que obtuvo una media 
inferior a 3 fue cree que es fácil hacer amigos en situaciones nuevas, posiblemente derivado de que el hecho de hacer amigos y de la facilidad o dificultad para la persona de hacerlo, no lo consideraban un aspecto sustancial ni relevante para la autodeterminación. Partiendo de que prácticamente todos los indicadores fueron altamente valorados (con medias superiores a 3), se ha intentado analizar aquellos que destacaban especialmente en las valoraciones, en contraste con los que eran valorados pero algo menos. Se han destacado aquellos con un valor medio superior a 3.75 (sobre 4) para indicar aquellos indicadores especialmente relevantes, frente a aquellos con valor medio inferior a 3.29, que siendo igualmente relevantes, parecían serlo menos.

Así, a continuación, se proporciona el porcentaje de respuestas para los indicadores valorados como más importantes por los participantes (aquellos con medias superiores a 3.75), haciendo referencia también a la dimensión de autodeterminación a la que pertenecen (Tabla 3). Resalta el hecho de que existan muchos ítems (concretamente 13 indicadores) en los que más del $80 \%$ de los participantes asignaron la puntuación máxima posible, destacando especialmente los indicadores decide cómo gastar el dinero y defiende sus derechos (en los que más del $90 \%$ asignan una puntuación de 4). Como se puede observar hay indicadores destacados en todas las dimensiones del constructo, indicando que los tres pilares en los que se fundamenta el constructo son igualmente importantes. Los indicadores más destacados que representan la dimensión "acciones volitivas" hacen referencia a tres componentes relacionados con esta dimensión: la autonomía de la persona en contextos sociales (cuando va a tiendas o bares, pide por sí mismo, o se comunica con familiares o amigos cuando quiere); el saber resolver problemas (si le duele algo o se hace una herida leve, sabe lo que tiene que hacer para resolverlo; o busca apoyo para conseguir sus objetivos cuando lo necesita) y, por último, las conductas volitivas relacionadas con el futuro personal (habla sobre cómo quiere que sea su vida o realiza actividades orientadas a la consecución de metas). Por su parte, los indicadores que más destacan dentro de la dimensión "acciones agenciales" se asocian a estrategias de autorregulación como la persistencia (es capaz de persistir para lograr sus objetivos), el establecimiento de metas (se plantea metas y objetivos en la vida) y la planificación (hace planes y decide qué hacer para lograr sus objetivos y plantea alternativas para alcanzar los objetivos, elige la que mejor se ajusta al objetivo). Por último, respecto a la dimensión "creencias de control-acción" destacan los indicadores relacionados con el autoconocimiento de alumno (y, específicamente, el conocimiento de las necesidades personales y las fortalezas); los relacionados con los derechos (tanto el conocimiento como la defensa de los mismos) y los relacionados con el empoderamiento (incluyendo la capacidad para expresarse libremente, pero también hacerlo con asertividad y respeto). 


\begin{tabular}{|c|c|c|c|c|c|c|}
\hline \multirow{2}{*}{ DIMENSIÓN } & \multirow{2}{*}{ Indicador } & \multicolumn{4}{|c|}{ IMPORTANCIA (\%) } & \multirow{2}{*}{ M (DT) } \\
\hline & & 1 & 2 & 3 & 4 & \\
\hline \multirow{8}{*}{$\begin{array}{l}\text { ACCIÓN } \\
\text { VOLITIVA }\end{array}$} & i029. Decide cómo gastar su dinero & 0 & 4.8 & 4.8 & 90.5 & $3.86(0.48)$ \\
\hline & $\begin{array}{l}\text { i008. Cuando va a tiendas o bares, pide (por sí mismo) lo que } \\
\text { quiere (con o sin apoyos) }\end{array}$ & 0 & 4.8 & 9.5 & 85.7 & $3.81(0.51)$ \\
\hline & i017. Realiza actividades que le gustan en su tiempo libre & 0 & 4.8 & 9.5 & 85.7 & $3.81(0.51)$ \\
\hline & $\begin{array}{l}\text { i024. Se comunica con sus amigos o familia cuando y como } \\
\text { quiere (sms, correos electrónicos o llamadas telefónicas) }\end{array}$ & 0 & 4.8 & 9.5 & 85.7 & $3.81(0.51)$ \\
\hline & $\begin{array}{l}\text { i015. Busca apoyo para conseguir sus objetivos cuando lo } \\
\text { necesita (en sus amigos, padres, profesionales, educadores, } \\
\text { etc.) }\end{array}$ & 0 & 0 & 19 & 81 & $3.81(0.40)$ \\
\hline & $\begin{array}{l}\text { i028. Realiza actividades (bien sea en el centro escolar, } \\
\text { ocupacional o laboral) orientadas a la consecución de metas } \\
\text { en el futuro (por ejemplo, obtener un empleo determinado o } \\
\text { mejorarlo) }\end{array}$ & 0 & 4.8 & 14.3 & 81 & $3.76(0.54)$ \\
\hline & i037. Habla sobre cómo quiere que sea su vida & 0 & 4.8 & 14.3 & 81 & $3.76(0.54)$ \\
\hline & $\begin{array}{l}\text { i010. Si le duele algo o se hace una herida leve, sabe lo que } \\
\text { tiene que hacer para resolverlo (incluyendo pedir ayuda) }\end{array}$ & 0 & 0 & 23.8 & 76.2 & $3.76(0.44)$ \\
\hline \multirow{4}{*}{$\begin{array}{l}\text { ACCIÓN } \\
\text { AGENCIAL }\end{array}$} & i056. Es capaz de ser persistente para lograr sus objetivos & 0 & 0 & 14.3 & 85.7 & $3.86(0.36)$ \\
\hline & i044. Se plantea metas y objetivos en la vida & 0 & 4.8 & 14.3 & 81 & $3.76(0.54)$ \\
\hline & $\begin{array}{l}\text { i055. Cuando plantea alternativas para alcanzar los objetivos, } \\
\text { elige la que mejor se ajusta al objetivo }\end{array}$ & 0 & 0 & 23.8 & 76.2 & $3.76(0.44)$ \\
\hline & i057. Hace planes y decide qué hacer para lograr sus objetivos & 0 & 0 & 23.8 & 76.5 & $3.76(0.44)$ \\
\hline \multirow{7}{*}{$\begin{array}{l}\text { CREENCIA } \\
\text { CONTROL- } \\
\text { ACCIÓN }\end{array}$} & i087. Identifica o conoce sus necesidades & 0 & 0 & 14.3 & 85.7 & $3.86(0.36)$ \\
\hline & i129. Defiende sus derechos & 0 & 5 & 5 & 90 & $3.85(0.49)$ \\
\hline & i127. Expresa lo que desea de forma asertiva & 0 & 0 & 19 & 76.2 & $3.80(0.41)$ \\
\hline & i085. Sabe qué cosas se le dan bien & 0 & 4.8 & 14.3 & 81 & $3.76(0.54)$ \\
\hline & i126. Conoce sus derechos & 0 & 5 & 15 & 80 & $3.75(0.55)$ \\
\hline & $\begin{array}{l}\text { i128. Les dice a sus amigos lo que quiere hacer cuando están } \\
\text { juntos }\end{array}$ & 0 & 0 & 25 & 75 & $3.75(0.44)$ \\
\hline & i130. Comunica su inconformidad con respeto & 0 & 0 & 25 & 75 & $3.75(0.44)$ \\
\hline
\end{tabular}

Fuente: elaboración propia.

Igualmente, es interesante comentar los indicadores valorados como relevantes, pero que obtienen medias inferiores a los anteriormente descritos (aquellos con medias inferiores a 3.29) y de nuevo haciendo referencia a la dimensión a la que pertenece cada uno (Tabla 4). 


\begin{tabular}{|c|c|c|c|c|c|c|}
\hline \multirow{2}{*}{ DIMENSIÓN } & \multirow{2}{*}{ Indicador } & \multicolumn{4}{|c|}{ IMPORTANCIA (\%) } & \multirow{2}{*}{ M (DT) } \\
\hline & & 1 & 2 & 3 & 4 & \\
\hline \multirow{7}{*}{$\begin{array}{l}\text { ACCIÓN } \\
\text { VOLITIVA }\end{array}$} & i018. Elige y escucha la música que le gusta & 9.5 & 9.5 & 23.8 & 57.1 & $3,29(1.01)$ \\
\hline & i016. Reconoce cuando se encuentra ante un problema & 0 & 23.8 & 23.8 & 52.4 & $3.29(0.85)$ \\
\hline & i021. Elige cómo se quiere peinar o cortar el pelo & 4.8 & 14.3 & 28.6 & 52.4 & $3.29(0.90)$ \\
\hline & $\begin{array}{l}\text { i022. Escoge los regalos para sus seres queridos (por ejemplo, } \\
\text { familiares, amigos, compañeros de trabajo) }\end{array}$ & 4.8 & 14.3 & 28.6 & 52.4 & $3.29(0.90)$ \\
\hline & $\begin{array}{l}\text { i033. Aprovecha las oportunidades que surgen para hacer } \\
\text { cosas nuevas }\end{array}$ & 4.8 & 9.5 & 42.9 & 42.9 & $3.24(0.83)$ \\
\hline & $\begin{array}{l}\text { i011. Llega puntual cuando tiene una cita (por ejemplo, con } \\
\text { amigos, su pareja, el médico, una clase o actividad laboral) }\end{array}$ & 4.8 & 19 & 33.3 & 42.9 & $3.14(0.91)$ \\
\hline & i025. Va a las cafeterías y restaurantes que le gustan & 0 & 23.8 & 42.9 & 33.3 & $3.10(0.77)$ \\
\hline \multirow{5}{*}{$\begin{array}{l}\text { ACCIÓN } \\
\text { AGENCIAL }\end{array}$} & i076. Se elogia cuando hace las cosas bien & 4.8 & 19 & 19 & 57.1 & $3.29(0.96)$ \\
\hline & i078. Explora distintas opciones para descubrir lo que le gusta & 9.5 & 9.5 & 23.8 & 57.1 & $3.29(1.01)$ \\
\hline & $\begin{array}{l}\text { i075. Cuando se enfrenta a una dificultad, hace lo que cree } \\
\text { que es mejor para él }\end{array}$ & 0 & 19 & 38.1 & 42.9 & $3.24(0.77)$ \\
\hline & $\begin{array}{l}\text { i074. Cuando hace las cosas, piensa en lo que es mejor para } \\
\text { él }\end{array}$ & 0 & 19 & 42.9 & 38.1 & $3.19(0.75)$ \\
\hline & $\begin{array}{l}\text { i077. Se concede premios cuando hace las cosas bien (por } \\
\text { ejemplo, se concede descansos, actividades agradables, } \\
\text { caprichos, etc.) }\end{array}$ & 0 & 28.6 & 28.6 & 42.9 & $3.14(0.85)$ \\
\hline \multirow{8}{*}{$\begin{array}{l}\text { CREENCIA } \\
\text { CONTROL- } \\
\text { ACCIÓN }\end{array}$} & i107. Cree que puede conseguir lo que quiera si trabaja duro & 0 & 23.8 & 23.8 & 52.4 & $3.29(0.85)$ \\
\hline & $\begin{array}{l}\text { i108. Cree que tienen capacidad para desempeñar el trabajo } \\
\text { que quiere }\end{array}$ & 4.8 & 14.3 & 28.6 & 52.4 & $3.29(0.90)$ \\
\hline & i112. Cree que puede hacer buenas elecciones & 4.8 & 9.5 & 38.1 & 47.6 & $3.29(0.85)$ \\
\hline & i088. Prueba cosas nuevas que cree que le van a gustar & 4.8 & 14.3 & 33.3 & 47.6 & $3.24(0.89)$ \\
\hline & i094. Siente que cae bien a otras personas & 0 & 14.3 & 52.4 & 33.3 & $3.19(0.68)$ \\
\hline & i095. Sabe que gusta a los demás & 0 & 28.6 & 38.1 & 33.3 & $3.05(0.80)$ \\
\hline & $\begin{array}{l}\text { 1102. Sabe que si se prepara, puede conseguir el trabajo que } \\
\text { quiere }\end{array}$ & 0 & 28.6 & 38.1 & 33.3 & $3.05(0.80)$ \\
\hline & $\begin{array}{l}\text { i080. Cree que le resulta fácil hacer amigos en situaciones } \\
\text { nuevas }\end{array}$ & 9.5 & 14.3 & 47.6 & 26.6 & $2.95(0.92)$ \\
\hline
\end{tabular}

Fuente: elaboración propia.

Para la dimensión "acciones volitivas" los indicadores que menos destacan son aquellos relacionados con los gustos y las elecciones en aspectos cotidianos de la vida (como por ejemplo ir a cafeterías, escuchar música, cortarse el pelo, elegir regalos o llegar puntual), en contraste con los más valorados que estaban más relacionados con metas de futuro o resolución de problemas. Los aspectos con menos importancia en la dimensión "acciones agenciales" resultaron ser los relacionados con tener en cuenta lo que le conviene a uno mismo antes de actuar (cuando se enfrenta a una dificultad, hace lo que cree que es mejor para él o cuando hace las cosas, piensa en lo que es mejor para él) y con el autorrefuerzo (se elogia cuando hace las cosas bien o se concede premios cuando hace las cosas bien). Por último, para la tercera dimensión los indicadores menos valorados fueron aquellos relativos con tener alta expectativas de éxito en el futuro (sabe 
que si se prepara, puede conseguir el trabajo que quiere, cree que puede conseguir lo que quiera si trabaja duro o cree que tiene capacidad para desempeñar el trabajo que quiere) y los asociados a sentirse valorado por otros (sabe que gusta a los demás o siente que cae bien a otras personas).

Además de indagar sobre el grado de importancia asignado por los participantes como grupo único, es igualmente interesante observar las diferencias existentes si tenemos en cuenta independientemente a profesionales, familias y personas con discapacidad. A nivel general, si consideramos la media general por grupos teniendo en cuenta todos los indicadores, los datos indican que los más críticos fueron los familiares $(\mathrm{M}=3.2$; $\mathrm{DT}=0.26)$; seguidos por los profesionales $(\mathrm{M}=3.59$; $\mathrm{DT}=0.36$ ); $\mathrm{y}$ las personas con discapacidad intelectual ( $M=3.79$; $D T=0.23$ ), es decir, los familiares fueron los que asignaron menor grado de importancia a los indicadores de autodeterminación, en contraste con los participantes con discapacidad intelectual que tendieron a dar puntuaciones más altas.

De hecho, los participantes con discapacidad intelectual asignaron puntuaciones altas a casi todos los indicadores, considerando que la mayoría de los elementos específicos en las tres dimensiones de autodeterminación eran muy importantes. En un $42 \%$ de los indicadores los cinco participantes con discapacidad intelectual coincidieron asignando la puntuación máxima de 4, mientras que en el grupo de familias no hubo ningún indicador en el que los seis familiares coincidieran asignando la puntuación máxima, y en el caso de los profesionales solo ocurrió en 5 de los 131 indicadores (menos de un 4 \%). Por eso, en el caso de la percepción de los participantes con discapacidad intelectual es más interesante fijarse en aquellos indicadores en los que alguno o varios de los participantes dieron una menor puntuación, de manera que podamos saber aquellos elementos menos importantes para ellos. Resulta muy llamativo que los ítems menos valorados correspondían casi en su totalidad a la tercera dimensión, y a los componentes relacionados con el empoderamiento, siendo los indicadores menos valorados por ellos los relacionados con usar la negociación como estrategia para convencer a los demás de lo que quiere $(M=3 ; D T=0.77)$ y con participar de forma activa en los grupos a los que pertenece $(M=3$; DT = 1.22).

En relación a las percepciones diferenciales entre los profesionales y las familias, se realizaron análisis no paramétricos de comparación de medias ( $u$ de Mann Whitney) para identificar aquellos indicadores en los que hubo diferencias significativas entre la importancia atribuida por profesionales y familias. Los resultados indican que hay ocho indicadores en los que los familiares otorgan una importancia significativamente menor, en contraste con la importancia dada por los profesionales (Tabla 5), destacando principalmente las diferencias entre los indicadores relacionados con las estrategias de planificación y autoevaluación para conseguir las metas y objetivos, o con la confianza y la persistencia para lograrlos a pesar de la opinión de los demás. 


\begin{tabular}{|c|c|c|c|c|}
\hline \multirow{2}{*}{ DIMENSIÓN } & \multirow{2}{*}{ Indicador } & Profesionales & Familiares & \multirow{2}{*}{$p$} \\
\hline & & M (DT) & M (DT) & \\
\hline \multirow{3}{*}{$\begin{array}{l}\text { ACCIÓN } \\
\text { VOLITIVA }\end{array}$} & i004. Ordena o recoge sus cosas & $3.50(0.71)$ & $2.67(0.52)$ & .042 \\
\hline & i021. Elige cómo se quiere peinar o cortar el pelo & $3.70(0.48)$ & $2.50(1.05)$ & .022 \\
\hline & $\begin{array}{l}\text { i034. Decide la hora a la que se levanta cuando no tiene } \\
\text { obligaciones }\end{array}$ & $3,50(0.85)$ & $2.50(0.84)$ & .042 \\
\hline \multirow{2}{*}{$\begin{array}{l}\text { ACCIÓN } \\
\text { AGENCIAL }\end{array}$} & i058. Sigue los pasos necesarios para alcanzar sus metas & $3.80(0.42)$ & $2.83(0.41)$ & .005 \\
\hline & i065. Compara sus resultados con las expectativas que tenía & $3.70(0.48)$ & $2,33(0.52)$ & .005 \\
\hline \multirow{3}{*}{$\begin{array}{l}\text { CREENCIAS } \\
\text { CONTROL- } \\
\text { ACCIÓN }\end{array}$} & $\begin{array}{l}\text { i080. Cree que les resulta fácil hacer amigos en situaciones } \\
\text { nuevas }\end{array}$ & $3.20(0.63)$ & $2.00(0.89)$ & .002 \\
\hline & $\begin{array}{l}\text { i115. Confía en sus capacidades para establecer sus metas } \\
\text { y objetivos }\end{array}$ & $3.90(0.32)$ & $3.00(0.63)$ & .022 \\
\hline & $\begin{array}{l}\text { i.122. Lleva a cabo sus propósitos aun cuando los demás } \\
\text { piensan que no es capaz de hacerlo }\end{array}$ & $3.90(0.32)$ & $2.83(0.75)$ & .011 \\
\hline
\end{tabular}

Fuente: elaboración propia.

En resumen, lo primero que muestran estos resultados es la importancia que tiene la autodeterminación en la vida de la persona con discapacidad intelectual, importancia que se ve reflejada en el alto valor que tienen cada uno de los indicadores observables (en definitiva, conductas, habilidades, creencias, actitudes...) que hacen a la persona convertirse en más autodeterminada. Además, todos los agentes que se encuentran en el entorno de una persona con discapacidad intelectual (incluida ella misma) otorgan un valor destacado a todos los indicadores. De hecho, aunque como se ha descrito se pueda observar un cierto patrón diferencial sobre la importancia atribuida por profesionales, familiares y personas con discapacidad, la realidad es que todos ellos otorgan valores muy altos a casi todos los indicadores.

\subsection{Discusión de los resultados sobre la importancia de la autodeterminación}

Todos estos resultados sobre la percepción que tienen profesionales, familias y personas con discapacidad intelectual sobre la importancia de la autodeterminación están en la línea de otros estudios existentes. Así, en un estudio realizado por Carter et al. (2013) en el que preguntaban a padres sobre la importancia de que su hijo aprendiera las habilidades relacionadas con la autodeterminación (autoconocimiento, autorregulación, hacer elecciones, resolver problemas, tomar decisiones, establecer metas y autodefensa) encontraron que las familias consideran importantes todos los componentes, sin destacar ninguno por encima del resto. Igualmente, otros estudios (Carter et al., 2008; Carter et al., 2015; Stang et al., 2009) también concluían que la mayoría de los profesionales encuestados, incluyendo profesores de escuela ordinaria, de especial o con otras funciones, consideraban como muy importantes habilidades como la resolución de problemas, la autorregulación, la toma de decisiones o el establecimiento de metas. Además, encontraron que los profesores de educación especial eran más sensibles y proporcionaban mayor importancia que los profesores de escuelas ordinarias en general (Stang et al., 2009) y en algunas de las habilidades relacionadas con empoderamiento (Carter et al., 2008). En relación a la percepción de las personas con discapacidad, Thoma 
y Getzel (2005) realizaron seis grupos de discusión con personas con diferentes discapacidades (pero sin discapacidad intelectual) y destacaron que componentes como la resolución de problemas, el establecimiento de objetivos, el autoconocimiento o la autogestión personal habían sido habilidades importantes para su éxito en contexto post-escolares. Resalta que, a diferencia con los datos aportados (aun teniendo en cuenta las limitaciones derivadas del tamaño de la muestra), no se han encontrado estudios que aborden conjuntamente la opinión de todos los agentes implicados.

En definitiva, es fundamental conocer y constatar que la autodeterminación es un pilar importante en la vida de las personas con discapacidad, y que tanto ellas como las personas de su entorno trabajen en la misma dirección, reconociendo y compartiendo los distintos puntos de vista y promoviendo aquellos aspectos más relevantes. En este sentido, igual que es relevante partir de la percepción sobre autodeterminación, es necesario poder disponer también de mejores recursos para apoyar el desarrollo de las habilidades relacionadas con la autodeterminación en el hogar, en los centros educativos y no educativos y en la comunidad (Carter et al., 2013; Carter et al., 2008).

\section{Retos y necesidades. ¿Qué necesidades tienen los profesionales de atención directa/familiares/ personas con discapacidad en relación con la promoción de la autodeterminación?}

Si bien las personas con discapacidad, sus familias y los profesionales que trabajan con ellas otorgan mucha importancia a la gran mayoría de indicadores o habilidades observables relacionadas con la autodeterminación, los retos y necesidades en cuanto a su promoción siguen vigentes. Además, por muy importante que sea la autodeterminación para la persona o sus allegados, transformar esta importancia en la creación de oportunidades de desarrollo de estas habilidades se erige como elemento clave (Carter et al., 2013). Por ello, resulta de gran importancia indagar en las perspectivas de familiares, profesionales y de las propias personas sobre los obstáculos y facilitadores que perciben, en definitiva, sobre sus necesidades a la hora de crear oportunidades para el aprendizaje de la autodeterminación y de actuar como agente causal de sus propias vidas, respectivamente.

Las propias personas con discapacidad reconocen que, en demasiadas ocasiones, los profesionales vetan el control sobre sus decisiones y aunque les puedan facilitar el acceso a los apoyos necesarios para actuar de forma autodeterminada, estos apoyos no se dan de forma continuada, limitando así las oportunidades para ejercer la autodeterminación (Nonnemacher y Bambara, 2011). En esta misma línea, las personas con discapacidad intelectual subrayan que en su relación con los profesionales hay actuaciones que dificultan su autodeterminación y otras que la facilitan (Nonnemacher y Bambara, 2011). Tomar decisiones por ellos, controlar sus gastos, estar inaccesible o tener una relación de poca confianza son algunos de los ejemplos que las propias personas con discapacidad destacan como obstáculos para ejercer la autodeterminación. Por contra, proporcionar oportunidades para vivenciar experiencias nuevas y tomar decisiones, una mayor accesibilidad y cercanía tanto de los educadores de atención directa como con otros profesionales de mayor nivel o especificidad; o escuchar las preferencias sin juzgar son algunas de las acciones que facilitan que dichas personas actúen de forma autodeterminada. 
A nivel familiar, las propias personas destacan también la necesidad de involucrar a los padres en el proceso de enseñanza/aprendizaje de la autodeterminación (Thoma y Getzel, 2005), a fin de que puedan promover en el hogar estrategias de autodeterminación, hecho que sigue siendo una tarea pendiente, al menos en nuestro contexto. Un estudio llevado a cabo en el ámbito español (Arellano y Peralta, 2013) destacó que casi la mitad de los familiares encuestados suelen escoger por sus hijos. A su vez, tampoco se suele fomentar, en contexto familiar, el autoconocimiento de la persona con discapacidad, ni la interacción con su medio, dado que no suelen tomar parte en las decisiones que atañen al hogar familiar (Arellano y Peralta, 2013). En la misma línea, una gran parte de los encuestados señaló que suelen presentar objetivos fáciles a sus hijos con discapacidad, evitando así la exposición al fracaso y la frustración.

Desde un enfoque a nivel macro y mesosistema, conocer las perspectivas de las personas y los profesionales puede ayudar a plantear necesidades a nivel de gestión de las instituciones proveedoras de apoyos para superar barreras en la promoción de la autodeterminación tales como la falta de apoyo administrativo y de continuidad en la implementación de estrategias o programas (Karvonen et al., 2004). Por ello, es fundamental conocer y operativizar vías factibles y efectivas de desarrollo de los profesionales sobre cómo implementar estrategias de promoción de la autodeterminación, siendo algunas de las más frecuentes (Carter et al., 2015): realizar talleres en el centro, consultar guías de buenas prácticas, establecer redes colaborativas entre profesionales, o buscar asesoramiento, entre otros. Dichas iniciativas, ayudarían a superar la principal limitación de los profesionales de atención directa que, si bien es cierto que consideran ciertas habilidades relacionadas con la autodeterminación como importantes, no suelen dedicarle tanto tiempo a su enseñanza como sería de esperar por la importancia atribuida (Carter et al., 2013).

En definitiva, parece innegable que, aunque son muchas las actuaciones que se realizan para promover la autodeterminación y que todos (profesionales, familiares y personas con discapacidad intelectual) atribuimos una importancia sustancial a este ámbito, existen aún grandes retos para promover la autodeterminación de las personas con discapacidad. Estos retos van desde aspectos muy concretos ligados a las relaciones interpersonales hasta cambios a mayor nivel asociados a recursos humanos, materiales e infraestructuras. Así, según mencionan Nonnemacher y Bambara (2011) el grado en que las personas con discapacidad actúan de forma autodeterminada parece estar mediado por la calidad de las relaciones interpersonales, concluyendo que la autodeterminación no se puede entender fuera de los contextos sociales donde las personas con discapacidad intelectual buscan y reciben apoyos. Sin embargo, sigue siendo igualmente necesario superar retos que limitan a las organizaciones; y garantizar que estas puedan disponer y propiciar contextos, recursos, actuaciones y apoyos orientados a promover la autodeterminación.

6. Conclusiones e implicaciones. ¿Qué interrogantes siguen existiendo y que necesitamos investigar para mejorar su comprensión?

A lo largo de este artículo son muchos los interrogantes que se han intentado responder. Tal y como se ha venido apuntando, queda mucho camino por recorrer y es necesario aumentar la investigación para ampliar el conocimiento en este ámbito (utilizando una variedad de metodologías: cuantitativas, cualitativas, estudios de caso...) y especialmente en nuestro contexto, donde el número de investigaciones es muy limitado. 
En primer lugar, surge la necesidad de conocer las creencias y aspectos culturales asociados a la autodeterminación que albergan profesionales, familiares y personas con discapacidad en contexto español, pues los conocimientos que tengan y la importancia que otorguen a la autodeterminación puede condicionar el grado en que se contemplan y trabajan los componentes específicos de la autodeterminación (e.g., toma de decisiones, resolución de problema) en los diferentes contextos (familiar y organizacional). El constructo de autodeterminación conlleva ciertos matices culturales que pueden condicionar su definición y promoción en función del marco cultural de referencia (Shogren, 2013), por lo que resulta necesario indagar en las creencias sobre autodeterminación que albergan profesionales y familiares de contextos concretos.

En segundo lugar, urge explorar las necesidades de los profesionales y familiares acerca de las dificultades u obstáculos con los que se enfrentan en sus contextos para crear oportunidades de ejercer la autodeterminación a las personas con discapacidad intelectual. Futuras investigaciones deberán aunar esfuerzos en conocer, indagar o profundizar en sus puntos de vista y su situación, con el fin de poder establecer mecanismos de apoyo para que profesionales y familiares de las personas con discapacidad dispongan de los recursos y conocimientos necesarios para promover la autodeterminación. Dicho conocimiento tiene el potencial de concretar y movilizar los recursos y apoyos específicos a familiares y profesionales para facilitar la enseñanza de la autodeterminación, brindando más oportunidades en casa, en la escuela, en la comunidad o en los contextos en los que se desenvuelva la persona, así como manteniendo las expectativas altas sobre el aprendizaje de la persona con discapacidad (Karvonen et al., 2004).

Así, identificar los componentes que priorizan las personas con discapacidad intelectual, las familias y los profesionales en relación a la autodeterminación puede ayudar a esos propios profesionales (de contextos educativos o no educativos) a conocer las áreas en las que focalizar un mayor énfasis y mejorar así, no solo la autodeterminación, sino también la transición a la vida adulta y el logro de objetivos personales deseados. Así mismo, podría ser igualmente necesario indagar si otros factores a nivel personal (edad, género, competencias...), familiar (cohesión, calidad de vida familiar,...) u organizacional (contexto escolar, ocupacional, laboral, tipo de profesionales,...) pueden estar mediando en la importancia y el énfasis por promover la autodeterminación. 


\section{Referencias bibliográficas}

Algozzine, B. et al. (2001): "Effects of interventions to promote self-determination for individuals with disabilities". Review of Educational Research, 71 (2): 219-277.

Arellano, A. y Peralta, F. (2013): "Self-determination of young children with intellectual disability: understanding parents' perspectives”. British Journal of Special Education, 40 (4): 175-181.

Carter, E. W. et al. (2015): "Administrator views on providing self-determination instruction in elementary and secondary schools". The Journal of Special Education, 49 (1): 52-64.

Carter, E. W. et al. (2013): "Self-determination among transition-age youth with autism or intellectual disability: Parent perspectives”. Research and Practice for Persons with Severe Disabilities, 38 (3): 129-138.

Carter, E. W. et al. (2008): "Promoting self-determination for transition-age youth: Views of high school general and special educators”. Exceptional Children, 75 (1): 55-70.

Cobb, B. et al. (2009): "Self-determination for students with disabilities: A narrative metasynthesis". Career Development for Exceptional Individuals, 32 (2): 108-114.

Díaz, Y. et al. (2013): "Guía didáctica PROYECTO V.I.D.A. Propuestas docentes para alumnado de secundaria con o sin discapacidad intelectual”. Madrid: FEAPS.

Field, S. y Hoffman, A. (1996): Steps to self-determination: A curriculum to help adolescents learn to achieve their goals. Austin, TX: Pro-Ed.

Field, S. y Hoffman, A. (2002): Lessons learned from implementing the steps to self-determination curriculum. Remedial and Special Education, 23 (2), 90-98.

Grupo de autogestores de ATZEGI (2009): Autodeterminación. Madrid: FEAPS.

Halpern, A.S. et al. (2000): NEXT S.T.E.P. Student Transition and Educational Planning (2nd ed.). Austin: PRO-ED.

Karvonen, M. et al. (2004): "Putting self-determination into practice". Exceptional Children, 71 (1): 23-41.

Luckasson, R. et al. (2002): Mental retardation. Definition, classification and systems of supports. Washington, DC: American Association on Mental Retardation [traducción al castellano de Verdugo, M. A. y Jenaro, C. (2004). Madrid: Alianza Editorial].

Luckasson, R. et al. (1992): Mental retardation. Definition, classification and systems of supports. Washington, DC: American Association on Mental Retardation [traducción al castellano de Verdugo, M. A. y Jenaro, C. (1997). Madrid: Alianza Editorial].

Martin, J. E. et al. (1996): ChoiceMaker self-determination curriculum. Longmont, CO: Sopris West.

Mumbardó-Adam, C. et al. (2018): "Psychometric properties of the Self-Determination Inventory: Student Report (spanish version): a structural equation modeling approach". American Journal on Intellectual and Developmental Disabilities, 123 (6), 545-557.

Mumbardó-Adam, C. et al. (2017): "Promoviendo la autodeterminación en el aula: el Modelo de Enseñanza y aprendizaje de la autodeterminación”. Siglo Cero, 48 (2): 41-59.

Nonnemacher, S. L. y Bambara, L. M. (2011). "I'm supposed to be in charge": Self-advocates' perspectives on their self-determination support needs". Intellectual and developmental disabilities, 49 (5): 327-340. 
Ponce, A. (2010): Formación en autodeterminación para familias. Madrid: FEAPS.

Raley, S. K. et al. (en prensa): "Curricula to teach skills associated with self-determination: A review of existing research". Education and Training in Autism and Developmental Disabilities.

Schalock, R. L. (1996): "Reconsidering the conceptualization and measurement of quality of life”, en Schalock, R. L. (ed.): Quality of life: Conceptualization and measurement. Washington, DC: American Association on Mental Retardation.

Schalock, R. y Verdugo, M.A. (2014): “Quality of life as a change agent”, en Brown, R. I. y Faragher, R. M. (eds.): Quality of Life and Intellectual Disability; Knowledge Application to Other Social and Educational Challenges. New York: Nova Science Publishers.

Shogren, K. A. (2013): "A social-ecological analysis of the self-determination literature". Intellectual and Developmental Disabilities, 51 (6): 496-511.

Shogren, K. A. et al. (2017): "Preliminary validity and reliability of scores on the Self-Determination InventoryStudent Report Version”. Career for Development and Transition for Exceptional Individuals, 40 (2): 92-103.

Shogren, K. A. et al. (2015): "Causal agency theory: reconceptualizing a functional model of self-determination". Education and Training in Autism and Developmental Disabilities, 50 (3): 251-263.

Shogren, K. A. et al. (2012): Effect of intervention with the self-determined learning model of instruction on access and goal attainment. Remedial and Special Education, 33 (5), 320-330.

Stang, K. K. et al. (2009): "Perspectives of general and special educators on fostering self-determination in elementary and middle schools". The Journal of Special Education, 43 (2): 94-106.

Thoma, C. A. y Getzel, E. E. (2005): "Self-determination is what it's all about": What Post-secondary Students with Disabilities Tell us are Important Considerations for Success". Education and Training in Developmental Disabilities, 40 (3): 234-242.

Verdugo, M. A. et al. (2015): Escala ARC-INICO de Evaluación de la Autodeterminación: Manual de aplicación y corrección. Salamanca: INICO.

Verdugo, M.A. y Schalock, R. L. (2010): “Últimos avances en el enfoque y concepción de las personas con discapacidad intelectual”. Siglo Cero, 41 (4): 7-21.

Vicente, E. et al. (2018): "What do stakeholders understand by self-determination? Consensus for its evaluation". Journal of Applied Research in Intellectual Disabilities, 32: 206-218.

Wehmeyer, M. L. (2003): “A funcional theory of self-determination: model overview”, en Wehmeyer, M. L. (eds.): Theory in self-determination: Foundations for educational practice. Springfield, IL: Charles C. Thomas.

Wehmeyer, M. L. (1999): “A functional model of self-determination: Describing development and implementing instruction". Focus on Autism and Other Developmental Disabilities, 14: 53-62.

Wehmeyer, M. L. et al. (2013): “Autodeterminación”, en Verdugo, M. A. y Schalock, R. L. (eds.): Discapacidad e inclusión. Manual para la docencia. Salamanca: Amarú.

Wehmeyer, M. L. et al. (2012): The impact of the self-determined learning model of instruction on student selfdetermination. Exceptional Children, 78 (2), 135-153.

Wehmeyer, M. L. et al. (2006): Escala de autodeterminación personal ARC Instrumento de valoración y guía de aplicación: Manual Técnico de la adaptación española. Madrid: CEPE. 
Wehmeyer, M. L. et al. (2000a): "A national survey of teachers' promotion of self-determination and studentdirected learning". Journal of Special Education, 34: 58-68.

Wehmeyer, M. L. et al. (2000b): "Promoting causal agency: The self-determined learning model of instruction". Exceptional Children, 66: 439-453.

Wehmeyer, M. L. et al. (1998): Teaching self-determination to students with disabilities. Basic skills for successful transition. Baltimore: Paul H. Brookes.

Wehmeyer, M. L. y Kelchner, K. (1995): The Arc's self-determination scale. Silver Springs, MD: The Arc of the United States. 